\title{
Landau-Zener-Stueckelberg effect in a model of interacting tunneling systems
}

\author{
D. A. Garanin \\ Institut für Physik, Johannes-Gutenberg-Universität, D-55099 Mainz, Germany
}

(Dated: November 16, 2018)

\begin{abstract}
The Landau-Zener-Stueckelberg (LZS) effect in a model system of interacting tunneling particles is studied numerically and analytically. Each of $N$ tunneling particles interacts with each of the others with the same coupling $J$. This problem maps onto that of the LZS effect for a large spin $S=N / 2$. The mean-field limit $N \rightarrow \infty$ corresponds to the classical limit $S \rightarrow \infty$ for the effective spin. It is shown that the ferromagnetic coupling $J>0$ tends to suppress the LZS transitions. For $N \rightarrow \infty$ there is a critical value of $J$ above which the staying probability $P$ does not go to zero in the slow sweep limit, unlike the standard LZS effect. In the same limit for $J>0$ LZS transitions are boosted and $P=0$ for a set of finite values of the sweep rate. Various limiting cases such as strong and weak interaction, slow and fast sweep are considered analytically. It is shown that the mean-field approach works well for arbitrary $N$ if the interaction $J$ is weak.
\end{abstract}

PACS numbers: 03.65.-w, 75.10.Jm

\section{INTRODUCTION}

The Landau-Zener-Stueckelberg (LZS) problem ${ }^{1,2,3}$ of quantum-mechanical transitions at an avoided level crossing induced by a linear-in-time energy sweep is well known in many areas of physics, in particular, in the physics of atomic and molecular collisions (see, e.g., Ref. 4). Recently the LZS effect was observed in crystals of single-molecule magnets and it was used to extract their tunneling level splittitng $\Delta .5,6$

The specifics of the LZS effect in solid-state systems is the macroscopically large number $N$ ot tunneling species such as spins of magnetic molecules in the above experiments ${ }^{5.6}$ These tunneling species are usually coupled with each other (mainly via the dipole-dipole interaction in the case of magnetic molecules) and the coupling energy $J$ can easily exceed the splitting $\Delta$. In this situation tunneling species (that can be described by pseudospins $S=1 / 2$ ) do not tunnel independently, and one has to consider the Schrödinger equation for the whole system that is described by $2^{N}$ variables. The latter is a new and tremendous problem since the whole Schrödinger equation becomes intractable even for a moderate number $N$ while analytical treatment is difficult because the system is far from the equilibrium.

As a plausible first step towards the solution of the LZS problem for a system of interacting particles one can consider a simplified model in which each particle interacts with all $N-1$ other particles with the same strength $J$, as was done in Ref. 7. For this model the Schrödinger equation simplifies so that one has to solve only $N+1$ equations instead of the $2^{N}$ equations in the general case. In the limit $N \rightarrow \infty$ the problem simplifies since the mean-filed approximation (MFA) becomes exact. The latter allows one to gain insights into the problem by analyzing its mean-field solution numerically as well as analytically in different limiting cases. Because the molecular field exerted on a tunneling particle from the others depends nonlinearly on time in the region of the level crossing, the problem cannot be linearized (c.f. Ref. 7) and the results differ essentially from the standard LZS solution. For instance, the ferromagnetic cou- pling, $J>0$, makes the energy sweep faster, and thus the probability $P$ to stay on the initial bare level increases. The nonlinear time dependence of the sweep within the MFA has stimulated Ref. 8, where the different kinds of sweep nonlinearities were investigated for a single tunneling system. Certainly the MFA for systems of interacting tunneling particles is a more complicated problem since in this case the form of the sweep is unknown from the beginning and it should be found self-consistently.

It is very interesting to study the difference between the exact quantum-mechanical and the mean-field solutions of the LZS problem for finite $N$. The original model maps of the model of a large spin $S=N / 2$ with the uniaxial anisotropy $D \sim J$, transverse field $H_{x} \sim \Delta$, and the sweeped longitudinal field $H_{z}(t)$. That is, the mean-field limit $N \rightarrow \infty$ of our model corresponds to the classical limit $S \rightarrow \infty$ for the effective large spin. In Ref. 7 it was shown numerically that for the model with $N=4$ and $J>0$ the exact and MFA results are qualitatively similar. It is very interesting, however, to investigate the problem for larger $N$ and for $J<0$.

Numerical and analytical study of these problems is the objective of this paper, the rest of which is organized as follows. In Sec. II the Hamiltonian is written down, the Schrödinger equation is simplified making use of the symmetry of the interaction, the problem is mapped onto that of a large spin and its general properties are studied. In Sec. III the case of well-separated resonances, $|J| \gg \Delta$ where the problem can be reduced to that of successive standard LZS transitions is investigated. In Sec. IV] the opposite case $J \ll \Delta$, where the problem can be solved perturbatively in $J$, is considered. Final results are worked out in the cases of fast sweep and slow sweep. In Sec. $\nabla$ general properties of the mean-field solution of the LZS problem with interaction are investigated, in particular, with the help of the mapping onto the classical-spin problem. Numerical solution shows, in particular, that for $J<0$ complete LZS transition, $P=0$, is achieved at some values of the sweep rate. Analytical treatment of the slow-sweep limit within the MFA is provided in Sec. VI 


\section{THE HAMILTONIAN}

We consider the model of $N$ double-level tunneling systems described by pseudospins $1 / 2$ and interacting each with each with an equal strength

$$
\widehat{H}=-\frac{1}{2} \sum_{i=1}^{N}\left[W(t) \sigma_{i z}+\Delta \sigma_{i x}\right]-\frac{J}{2} \sum_{i \neq j=1}^{N} \sigma_{i z} \sigma_{j z} .
$$

Here $\boldsymbol{\sigma}_{i}$ are Pauli matrices, $W(t)$ is the energy sweep that is taken to be linear in time

$$
W(t) \equiv E_{-1}(t)-E_{1}(t)=v t,
$$

$\Delta$ is the level splitting at resonance in the absence of interaction $(t=0$ and $J=0)$, whereas $J$ is the interaction constant. The case $J>0$ corresponds to the ferromagnetic (FM) coupling, whereas that of $J<0$ corresponds to the antiferromagnetic frustrating (AFMF) coupling. In the latter case, the ground state of the system with $W=\Delta=0$ is a highly degenerate state with the minimal total spin. If $J=0$, the problem simplifies to the well known LZS problem for individual tunneling systems. If these system at $t \rightarrow-\infty$ are in the bare ground state $\psi_{-1} \equiv|\downarrow\rangle$ before crossing the resonance, the probability to stay in this state after crossing the resonance at $t \rightarrow \infty$ is given by ${ }^{2.3 .9 .10}$

$$
P(\infty) \equiv P=e^{-\varepsilon}, \quad \varepsilon \equiv \frac{\pi \Delta^{2}}{2 \hbar v}
$$

(Probabilities without the argument are shortcuts for the final-state probabilities at $t=\infty$ throughout the paper). For the problem with interaction, the wave function of the system can be written as the expansion over the direct-product states

$$
\begin{aligned}
& \Psi(t)=\sum_{m_{1}, \ldots, m_{N}=-1,1} C_{m_{1}, \ldots, m_{N}}(t) \psi_{m_{1}} \otimes \ldots \otimes \psi_{m_{N}} \\
& \psi_{-1}=\left(\begin{array}{l}
0 \\
1
\end{array}\right)=|\downarrow\rangle, \quad \psi_{1}=\left(\begin{array}{l}
1 \\
0
\end{array}\right)=|\uparrow\rangle .
\end{aligned}
$$

The initial condition for $\Psi(t)$ is $C_{-1, \ldots,-1}(-\infty)=1$ whereas all other coefficients are zero, i.e., the system starts in the state with all spins down. With time the state of the system becomes a superposition of all possible basis states in Eq. (4). The one-particle probability to remain in the initial state -1 for our $N$-particle system is given by

$$
P_{N}(t)=\sum_{m_{2}, \ldots, m_{N}=-1,1}\left|C_{-1, m_{2}, \ldots, m_{N}}(t)\right|^{2}
$$

and it starts from $P_{N}(-\infty)=1$.

The solution of the LZS problem for this model is simplified by the fact that the coefficients $C_{m_{1}, \ldots, m_{N}}(t)$ depend only on the number $k$ of spins up while they are independent on the choice of these spins. Thus one can label the states by the index $k$ only that results in the Schrödinger equation

$$
i \hbar \dot{C}_{k}=E_{k}(t) C_{k}-\frac{k \Delta}{2} C_{k-1}-\frac{(N-k) \Delta}{2} C_{k+1}
$$

for $k=0, \ldots, N$ and with the bare energies

$$
E_{k}(t)=\left(\frac{N}{2}-k\right) W(t)+2 J k(N-k)+\text { const. }
$$

Each $k$-state is realized by $N ! /[(N-k) ! k !]$ different choices of the indices $m_{1}, \ldots, m_{N}$ in Eq. (4). For $J<0$, the ground state with $W=\Delta=0$ corresponds to $k=N / 2$ and is highly degenerate. In Eq. (5) $m_{1}$ is fixed to -1 , and the remaining indices $m_{2}, \ldots, m_{N}$ can also be parametrized by $k$. In the corresponding number of realizations, one should use $N-1$ instead of $N$ that leads to

$$
P_{N}(t)=\sum_{k=0}^{N-1} \frac{(N-1) !}{(N-1-k) ! k !}\left|C_{k}(t)\right|^{2} .
$$

It is convenient to introduce the coefficients

$$
c_{k} \equiv \sqrt{\frac{N !}{(N-k) ! k !}} C_{k}
$$

that satisfy the normalization condition $1=\sum_{k=0}^{N}\left|c_{k}\right|^{2}$ and the Schrödinger equation

$$
i \hbar \dot{c}_{k}=E_{k}(t) c_{k}-\frac{\Delta}{2} l_{k-1, k} c_{k-1}-\frac{\Delta}{2} l_{k, k+1} c_{k+1}
$$

with $l_{k, k+1} \equiv \sqrt{(N-k)(k+1)}$. Then Eq. (8) simplifies to

$$
P_{N}(t)=\sum_{k=0}^{N}\left(1-\frac{k}{N}\right) p_{k}(t), \quad p_{k}(t) \equiv\left|c_{k}(t)\right|^{2} .
$$

Eq. (10) maps on the corresponding Schrödinger equation for a large pseudospin $S=N / 2$ with the Hamiltonian

$$
\widehat{H}_{S}=-H_{z}(t) S_{z}-H_{x} S_{x}-D S_{z}^{2}+\text { const },
$$

where

$$
H_{z}(t)=W(t), \quad H_{x}=\Delta, \quad D=2 J
$$

(we set $g \mu_{B}=1$ so that the "magnetic field" is energy dimensional). Note that the excitation number $k$ is related to the eigenvalue $m$ of $S_{z}$ by $k=N / 2+m$. Thus $P(t)$ maps onto

$$
P_{N}(t)=\frac{1}{2}\left(1-\frac{\left\langle S_{z}\right\rangle_{t}}{S}\right) .
$$

The large-spin model defined by Eq. (12) is well known from the physics of the single-molecule magnet $\mathrm{Mn}_{12}$. For small $\Delta$ the energy levels of the system as function of $W$ are nearly straight lines with avoided crossings (see Fig. 1). Splittings at avoided crossings are or order $\Delta(\Delta / J)^{n}$, where $n$ are appropriate powers 11

Our model described by Eqs. (1) or (12), although not completely realistic, allows one to trace down the influence of the interaction on the transition probabilities and to obtain a number of interesting analytical results. We 

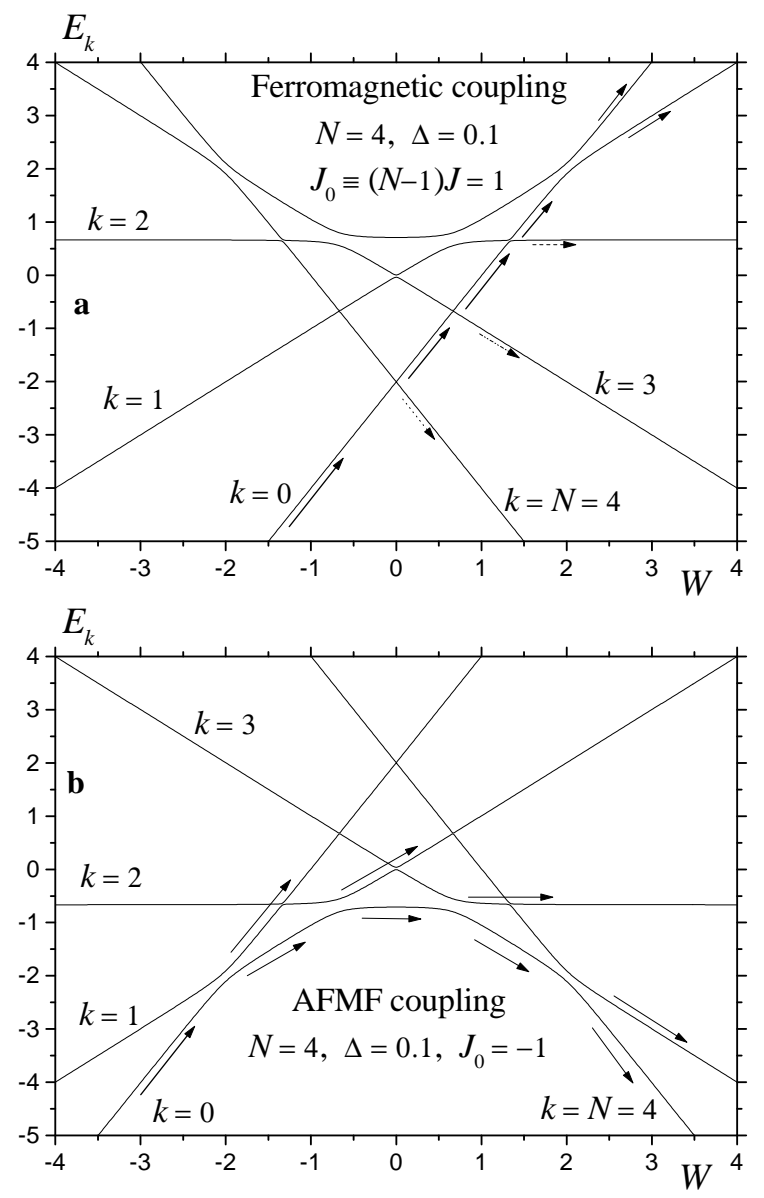

FIG. 1: $a$-Exchange-split resonances for a ferromagnetically coupled spin cluster for $|J| \gtrsim \Delta$. Transitions shown by the dashed and dotted arrows arise in higher orders in $\Delta / J$ and are much weaker. $b$-Same for an AFMF coupled spin cluster.

will see that, in particular, suppression of transitions for the ferromagnetic interactions $(J>0)$ can be easily understood. An advantage of this simplified model is the possibility to solve the problem numerically up to much larger system sizes, compared to models with realistic interactions. For the latter, the number of different coefficients in Eq. (4) and thus of differential equations to solve is $2^{N}$, whereas in our case there are only $N+1$ equations.

For numerical calculations we use Wolfram Mathematica 4.0 that employs, in particular, a very accurate differential equation solver needed for handling strongly oscillating solutions over large time intervals. The results of solving Eq. (10) are shown in Fig. 2 for a cluster of three ferromagnetically coupled particles.

\section{WELL-SEPARATED RESONANCES}

The interaction can profoundly influence the LZS effect if it is strong enough, $|J| \gtrsim \Delta$. The general tendency can be already seen from the well-known mean-field argument. If one of the tunneling particles flips to another

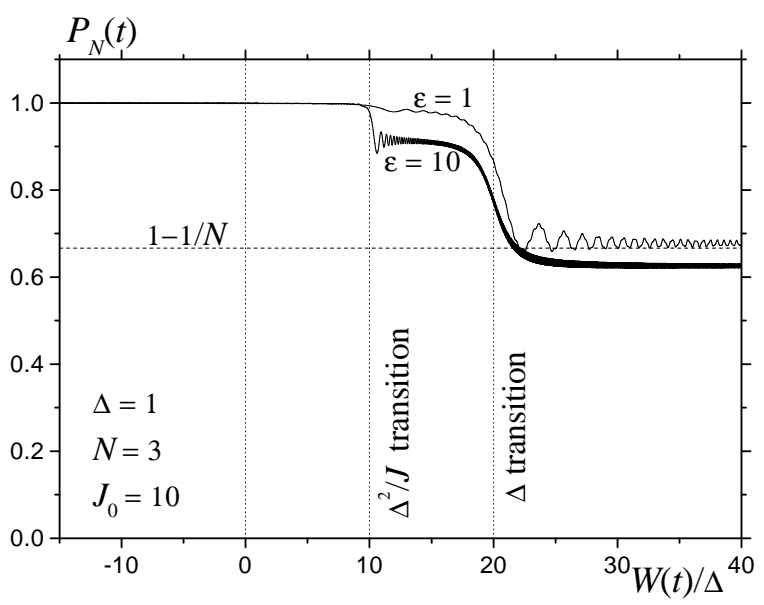

FIG. 2: Time dependence of the one-particle staying probability $P_{N}$ as function of the energy sweep $W(t)$ for a system of $N=3$ ferromagnetically coupled particles. At intermediate sweep rate, $\varepsilon=1$ only the first-order LZS transition at $W=2 J_{0}$ (the $\Delta$ transition) is seen. For rather slow sweep rates (here $\varepsilon=10$ ), higher-order transitions (here the $\Delta^{2} / J$ transition at $W=0$ ) are switching on.

bare state during the resonance crossing, then the total field (the external sweep field plus the molecular field) on all other particles jumps. For the ferromagnetic coupling, $J>0$, the jump of the total field is positive, other particles are brought past the resonance and lose their chance to flip, and thus transition probability is suppressed. For the antiferromagnetic frustrating coupling, the jump of the total field is negative and other particles are being set back before the resonance and are getting one more chance to cross the resonance at some larger field value and flip; Thus the transition probability for the system should increase.

The influence of the interaction can be readily illustrated within a rigorous many-body quantum language for our model if one considers the energy levels of the system shown in Fig. [1] One can see that instead of a single resonance at $W=0$ for individual or noninteracting particles there is an interaction-split resonance that consists of many avoided line crossings. These avoided line crossings are well separated from each other on the plot if $|J| \gg \Delta$. The question whether these well-separated crossings can be considered as a succession of independent LZS transitions (i.e., whether they are dynamically well separated) depends on the sweep rate. Whereas for the slow sweep, $\varepsilon \gtrsim 1$ [see Eq. (3)] the condition $|J| \gg \Delta$ is sufficient, for the fast sweep, $\varepsilon \lesssim 1$, a more stringent condition is required that follows from Eq. (16) of Ref. 8. The resulting combined condition for the dynamically well-separated resonances thus would be

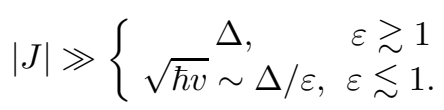

This is, however, only an apriori estimation considering two resonances. For $N \gg 1$ there are many resonances, and the deviations from the single-resonance picture can 
accumulate with the increase of $N$. One can do an aposteriori estimation in the slow-sweep limit where the result for the well-separated resonances $P \rightarrow 1-1 / N$ for $\varepsilon \rightarrow \infty$ following from Eq. (20) as well as the mean-field result for $N \gg 1$ and non-separated resonances [the first line of Eq. (92)] are available, in both cases $1-P \ll 1$. The crossover between these results should take place at $|J| \sim$ $\Delta N^{1 / 2}$, thus the first line of Eq. (15) should be replaced by

$$
|J| \gg \Delta N^{1 / 2}, \quad \varepsilon \gtrsim 1
$$

The second line of Eq. (15) could be also modified by $N$ but it is difficult to derive an exact condition.

A perturbative analysis of the stationary Schrödinger equation for our model shows that tunnel level splitting of the bare levels with different values of $k$ has the form ${ }^{11}$

$$
\delta E_{k_{1}, k_{2}} \sim \Delta\left(\frac{\Delta}{J}\right)^{\left|k_{1}-k_{2}\right|-1} .
$$

Thus for $|J| \gg \Delta$ only the first-order or direct transitions with $\left|k_{1}-k_{2}\right|=1$ should be taken into account whereas the higher-order transitions are weak (see Fig. (1).

Now it becomes clear that for the ferromagnetic coupling and well-separated resonances only the strong transition between the initial level $k=0$ and the level $k=1$ happens. That is, in another language, only one particle of $N$ has a chance to tunnel, and the tunneling probability for the whole system is strongly reduced. For a quantitative analysis one can neglect all $c_{k}$ with $k>1$ in Eq. (10) that yields the system of equations

$$
\begin{aligned}
& i \hbar \dot{c}_{0}=E_{0} c_{0}-\frac{\Delta}{2} \sqrt{N} c_{1} \\
& i \hbar \dot{c}_{1}=E_{1} c_{1}-\frac{\Delta}{2} \sqrt{N} c_{0}
\end{aligned}
$$

that maps on the standard LZS problem with $\Delta \Rightarrow \Delta_{N} \equiv$ $\Delta \sqrt{N}$ and with the resonance at $W=2(N-1) J$ instead of $W=0$. The final-state probabilities for Eq. (18) are according to Eq. (3)

$$
p_{0}=P^{N}=e^{-N \varepsilon}, \quad p_{1}=1-P^{N} .
$$

Then with the help of Eq. (11) the one-particle staying probability for $J>0$ can be cast into the form

$$
P_{N}=1-\frac{1}{N}\left(1-P^{N}\right)
$$

and it varies between 1 in the fast-sweep limit and $1-1 / N$ in the slow-sweep limit. Thus suppression of transitions by the ferromagnetic coupling is extremely strong for a large number of particles $N$. Expansion of the transition probability of Eq. (20) in the fast-sweep limit reads

$$
1-P_{N} \cong \varepsilon-\frac{N}{2} \varepsilon^{2}+O\left(\varepsilon^{3}\right), \quad(\varepsilon \ll 1 / N) .
$$

Note that the first term of this expansion is insensitive to the interaction (in this context to the number of interacting particles) and is the same as for the standard LZS problem, $1-P=1-e^{-\varepsilon} \cong \varepsilon-\varepsilon^{2} / 2 !+\varepsilon^{3} / 3 !-\ldots$.

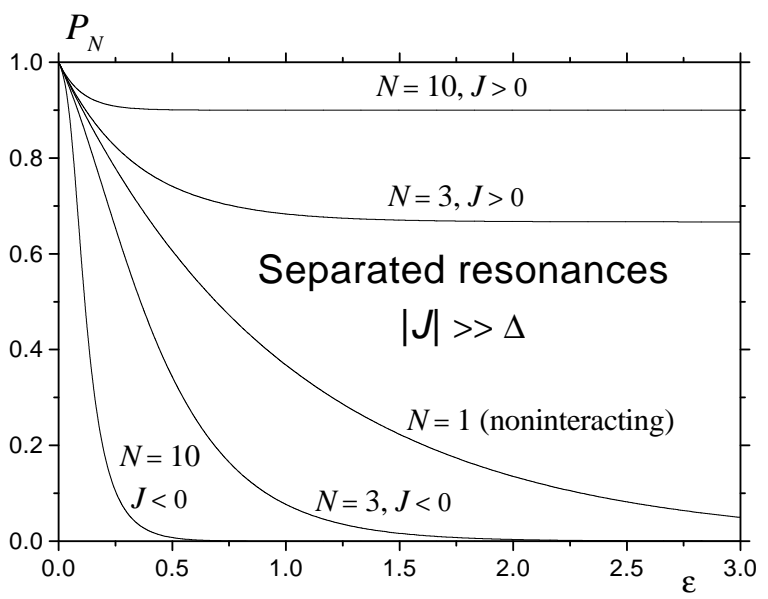

FIG. 3: One-particle staying probability $P_{N}$ vs the sweeprate parameter $\varepsilon$ for systems with FM and AFMF interaction in the case of well separated resonances.

The AFMF coupling $J<0$ favors transitions, as can be seen from Fig. 10. The original resonance is splitted by the interaction into $N$ resonances filling equidistantly the range $-2(N-1) J \leq W \leq 2(N-1) J$. One can see that in the limit of slow sweep the system remains on the lowest adiabatic energy level thus $P_{N} \rightarrow 0$, in contrast to the ferromagnetic case. For well-separated resonances the problem described by Eq. (10) splits into independent standard LZS problems for the resonances between the levels $k$ and $k+1$ that are described by an effective splitting $\Delta_{N, k}=\Delta l_{k, k+1}$ [cf. Eq. (18)]. It is convenient to designate the probability to stay in state $k$ after crossing with state $k-1$ but before crossing with state $k+1$ (see Fig. 10) as $p_{k}$ (mid). Then solutions of the LZS problems for individual crossings along with conditions of the probability conservation can be written as

$$
\begin{aligned}
p_{k}(\infty) & =P^{(N-k)(k+1)} p_{k}(\mathrm{mid}) \\
p_{k}(\mathrm{mid}) & =p_{k-1}(\mathrm{mid})-p_{k-1}(\infty) \\
p_{k-1}(\infty) & =P^{(N-k+1) k} p_{k-1}(\mathrm{mid}),
\end{aligned}
$$

etc. These can be combined into the recurrence relation

$$
\begin{aligned}
p_{k}(\infty) & =P^{(N-k)(k+1)}\left(P^{-(N-k+1) k}-1\right) p_{k-1}(\infty) \\
& =P^{N-2 k}\left(1-P^{(N-k+1) k}\right) p_{k-1}(\infty)
\end{aligned}
$$

that has to be iterated with the initial condition $p_{0}(\infty)=$ $P^{N}$. In the slow-sweep limit $\varepsilon \gg 1 / N$, one has $P^{N} \ll 1$, thus one can drop the factor $\left(1-P^{(N-k+1) k}\right) \cong 1$ in the second line of Eq. (23) that after iteration results in $p_{k} \cong$ $P^{(N-k)(k+1)}$. In this case, in Eq. (11) $p_{0} \cong p_{N-1} \cong P^{N}$ are dominant for large $N$ whereas all other summands are much smaller. This yields

$$
P_{N} \cong\left(1+\frac{1}{N}\right) P^{N} \ll 1, \quad(\varepsilon \gg 1 / N)
$$

for the antiferromagnetic coupling. In the fast-sweep limit the solution of Eq. (23) can be expanded in powers of $\varepsilon$, after which Eq. (11) yields for the transition 


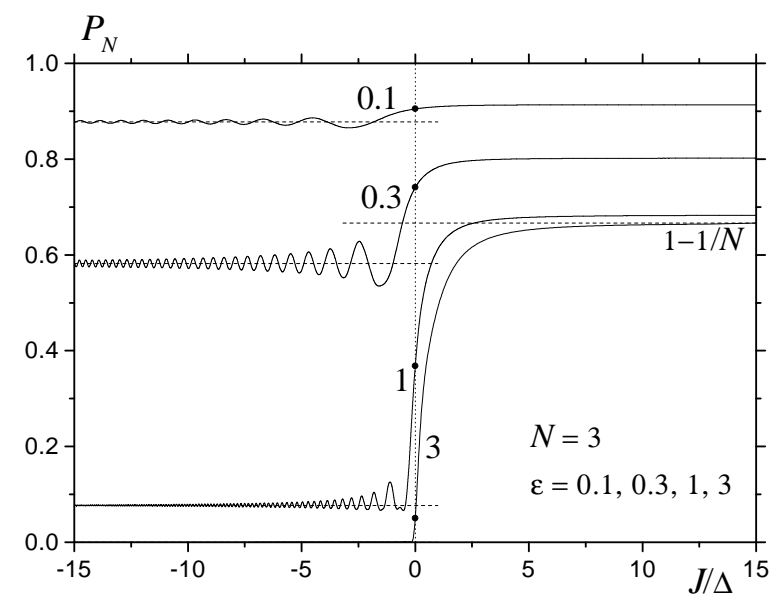

FIG. 4: Numerically computed staying probability $P_{N}$ vs $J / \Delta$ for $N=3$ and different sweep rate parameters $\varepsilon$. The horizontal dashed lines on the left side of the plot are asymptotes corresponding to well-separated resonances.

probability

$$
1-P_{N} \cong \varepsilon+\left(\frac{3 N}{2}-2\right) \varepsilon^{2}+O\left(\varepsilon^{3}\right), \quad(\varepsilon \ll 1 / N) .
$$

Again, the first term of this expansion is the same as in the standard LZS problem. For intermediate sweep rates one can iterate Eq. (23) numerically and substitute the solution for $p_{k}$ into Eq. (11). The resulting curves $P_{N}$ vs $\varepsilon$ are shown in Fig. 3 along with those for the ferromagnetic coupling, Eq. (20).

To conclude this section, we show the numerically computed dependences of $P_{N}$ on $J / \Delta$ for $N=3$ and different $\varepsilon$ in Fig. 4. The case of well-separated resonances that was considered in this section corresponds to the plateaus on the left and on the right sides of the plot.

\section{FAST SWEEP AND WEAK COUPLING}

In the preceding section we have considered the strongcoupling limit $|J| \gg \max \left(\Delta N^{1 / 2}, \Delta / \sqrt{\varepsilon}\right)$ in which individual resonances are well separated from each other and one thus deals with successive standard LZS transitions. The opposite limiting case is that of the weak coupling $J$. This case can be solved by a perturbative expansion in $J$ with the standard LZS solution as the zeroth-order appriximation. The situation further simplifies in the fastsweep limit, where the transition probability is small and the coefficients $c_{k}$ in Eq. (10) decrease with $k$ as powers of $\varepsilon \ll 1$. In particular, $c_{0}$ contains terms of orders $\varepsilon^{0}, \varepsilon^{1}$, and $\varepsilon^{2}$, the coefficient $c_{1}$ contains $\varepsilon^{1 / 2}$ and $\varepsilon^{3 / 2}$, the coefficient $c_{2}$ starts with $\varepsilon$, etc. Knowing these contributions into $c_{0}, c_{1}$, and $c_{2}$ is sufficient to set up the expansion of $P_{N}$ up to the first nontrivial order $\varepsilon^{2}$. A straighforward but cumbersome calculation yields

$$
1-P_{N} \cong \varepsilon-\left(\frac{1}{2}+\frac{4 J_{0}}{\sqrt{2 \pi \hbar v}}\right) \varepsilon^{2}+O\left(\varepsilon^{3}\right)
$$

$$
=\varepsilon-\frac{1}{2} \varepsilon^{2}-\frac{4 J_{0}}{\Delta} \varepsilon^{5 / 2}+\ldots
$$

where we have defined

$$
J_{0} \equiv(N-1) J
$$

One can see, again that ferromagnetic interaction suppresses transitions whereas the AFMF interaction facilitates transitions, and that the effect of interaction is increased by the number particles in the system. In fact, however, in our model the interaction should scale, on physical grounds, with the system's size, i.e., $J_{0} \equiv$ $(N-1) J$ should be size independent. Note that in the fast-sweep limit one could do the expansion in $\varepsilon$ for arbitrary $J$. This leads, however, to cumbersome multiple integrals. The strong-coupling limit for the fast sweep $\varepsilon \ll 1$ has been considered above, and the corresponding counterparts of Eq. (26) for ferro-and antiferromagnetic coupling are Eqs. (21) and (25).

It should be noted that although Eq. (26) is valid for $\varepsilon \ll 1$, the method of its derivation above is only justified for $\varepsilon \ll 1 / N$, i.e. for much faster sweep rates, if $N \gg 1$. Indeed, in the course of the derivation the terms of orders $(\varepsilon N)^{1 / 2}, \varepsilon N$, etc., appear that are required to be small. Only at the very end the leading $N$ contributions cancel each other that leads to Eq. (26) that fortunately has a larger applicability range. In fact, for large systems with weak interaction the most populated final states are

$$
k_{\max }=N(1-P) \cong N \varepsilon,
$$

so that keeping only the states with $k=0,1,2$ above was not justified for $N \varepsilon \gtrsim 1$. Eq. (28) can be easily derived for an assembly of noninteracting tunneling species. To this end, we use the coefficients $C_{k}$ of Eq. (6) and represent them in the factorized form

$$
C_{k}=a_{-1}^{N-k} a_{1}^{k},
$$

where $a_{-1}$ and $a_{1}$ are the coefficients of the wave function of the one-particle problem, $\psi=a_{-1} \psi_{-1}+a_{1} \psi_{1}$. This yields

$$
\left|C_{k}\right|^{2}=\left|a_{-1}\right|^{2(N-k)}\left|a_{1}\right|^{2 k}=P^{N-k}(1-P)^{k}
$$

that can be plugged into Eq. (8) to give

$$
P_{N}=\sum_{k=0}^{N-1} \frac{(N-1) !}{(N-1-k) ! k !} P^{N-k}(1-P)^{k} .
$$

The summand in this formula has its maximum at $k=$ $k_{\text {max }}$ given by Eq. (28) and it becomes a narrow Gaussian packet around $k_{\max }$ for $N \gg 1$.

A more rigorous method of handling the fast-sweep and weak coupling limits uses the one-particle density matrix that is defined by

$$
\rho_{-1,-1}=P_{N}, \quad \rho_{1,1}=1-\rho_{-1,-1}=1-P_{N}
$$

with $P_{N}$ of Eq. (8) and

$$
\rho_{1,-1}=\sum_{m_{2}, \ldots, m_{N}=-1,1} C_{1, m_{2}, \ldots, m_{N}}^{*} C_{-1, m_{2}, \ldots, m_{N}}
$$




$$
\begin{aligned}
& =\sum_{k=0}^{N-1} \frac{(N-1) !}{(N-1-k) ! k !} C_{k+1}^{*} C_{k} \\
\rho_{-1,1} & =\rho_{1,-1}^{*} .
\end{aligned}
$$

The density-matrix equation (DME) can be obtained by time differentiating of $\rho_{m n}$ and employing Eq. (6) that yields

$$
\begin{aligned}
& \hbar \dot{\rho}_{-1,-1}=\frac{i \Delta}{2}\left(\rho_{-1,1}-\rho_{1,-1}\right)=\Delta \operatorname{Im} \rho_{1,-1} \\
& \hbar \dot{\rho}_{1,-1}=-i W(t) \rho_{1,-1}+\frac{i \Delta}{2}\left(\rho_{1,1}-\rho_{-1,-1}\right) \\
& +2 i J \sum_{k=0}^{N-1} \frac{(N-1) !}{(N-1-k) ! k !}(N-2 k-1) C_{k} C_{k+1}^{*}
\end{aligned}
$$

In the absence of interaction, $J=0$, the last term in $\dot{\rho}_{-1,1}$ disappears and one obtains a DME for one isolated particle that is equivalent to the one-particle Schrödinger equation. Note that solving this one-particle equation in the fast-sweep limit requires $\varepsilon \ll 1$, in contrast to Eq. (6) with $J=0$ that requires $\varepsilon \ll 1 / N$.

For $J \neq 0$ Eqs. (34) do not form a closed system of equations. In this case Eqs. (34) can only be useful if an approximation for the interaction term be found. In particular, one can consider the interaction term perturbatively in $J$ and use

$$
\rho_{m n}=\rho_{m n}^{(0)}+\delta \rho_{m n}
$$

where $\rho_{m n}^{(0)}$ is the density matrix without interaction that satisfies

$$
\begin{aligned}
& \hbar \dot{\rho}_{-1,-1}^{(0)}=\Delta \operatorname{Im} \rho_{1,-1}^{(0)} \\
& \hbar \dot{\rho}_{1,-1}^{(0)}=-i W(t) \rho_{1,-1}^{(0)}+i \Delta\left(\frac{1}{2}-\rho_{-1,-1}^{(0)}\right)
\end{aligned}
$$

and $\delta \rho_{m n}$ is the correction term. The latter satisfies equations similar to Eqs. (34) in which, however, $C_{k} C_{k+1}^{*}$ is replaced by its noninteracting-particle expression following from Eq. (29),

$$
\begin{aligned}
& C_{k} C_{k+1}^{*}=a_{-1}^{N-k} a_{1}^{k}\left(a_{-1}^{*}\right)^{N-k-1}\left(a_{1}^{*}\right)^{k+1} \\
& \quad=\left|a_{-1}\right|^{2(N-k-1)}\left|a_{1}\right|^{2 k} a_{-1} a_{1}^{*} \\
& \quad=\left(\rho_{-1,-1}^{(0)}\right)^{N-k-1}\left(1-\rho_{-1,-1}^{(0)}\right)^{k} \rho_{-1,1}^{(0)} .
\end{aligned}
$$

With the use of the latter, the sum over $k$ in Eqs. (34) can be performed to yield

$$
\begin{aligned}
& \hbar \delta \dot{\rho}_{-1,-1}=\Delta \operatorname{Im} \delta \rho_{1,-1} \\
& \hbar \delta \dot{\rho}_{1,-1}=-i W(t) \delta \rho_{-1,1}-i \Delta \delta \rho_{-1,-1} \\
& \quad-2 i J_{0}\left(1-2 \rho_{-1,-1}^{(0)}\right) \rho_{1,-1}^{(0)} .
\end{aligned}
$$

Solving Eqs. (36) and (38) perturbatively for $\varepsilon \ll 1$ results in Eq. (26).

In accord with the remark at the beginning of this section, $J$-perturbative Eqs. (36) and (38) can be solved in terms of the hypergeometric functions for any $\varepsilon$ (For the

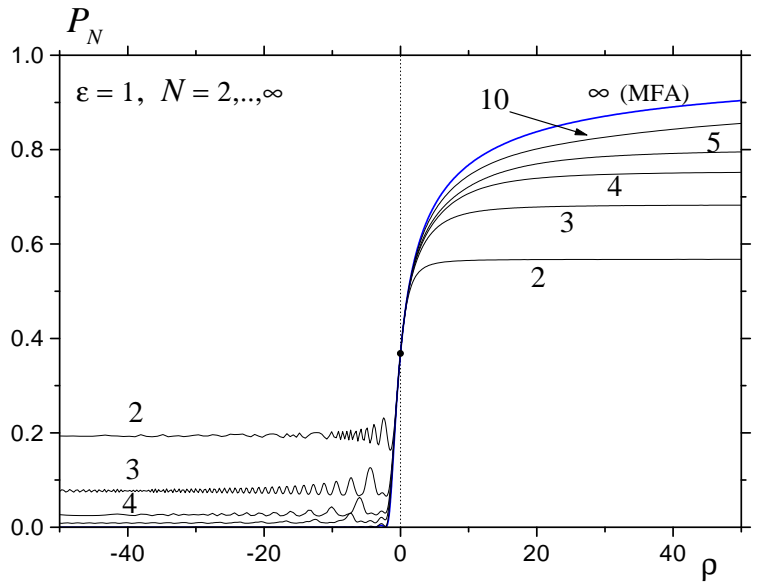

FIG. 5: Numerically computed staying probability $P_{N}$ vs $\rho \equiv 2 J_{0} / \Delta$ for $\varepsilon=1$ and different system sizes $N$, including the mean-field result for $N \rightarrow \infty$.

standard LZS problem, $J=0$, this was done by Zener ${ }^{2}$ ) It can be shown that in the slow-sweep limit this solution simplifies to

$$
\left.\frac{d P}{d \rho}\right|_{\rho=0}=c \varepsilon e^{-\varepsilon}, \quad \varepsilon \gg 1, \quad \rho \equiv \frac{2 J_{0}}{\Delta},
$$

where $c$ is a number and the parameter $\rho$ should not be confused with the density matrix.

\section{THE MEAN FIELD APPROXIMATION}

If each of tunneling particles interacts with all the other particles with a coupling of the same sign, as is the case in our model for $N \gg 1$, then the mean-field approximation (MFA) should work well. The MFA employs a one-particle density-matrix equation or a one-particle Schrödinger equation in which the interaction enters via the molecular field. For the model of Eq. (11) one has $W(t) \Rightarrow W_{\text {eff }}(t)$ where

$$
W_{\text {eff }}(t)=W(t)+2 J_{0}\left(1-2 \rho_{-1,-1}\right)
$$

in the DME of Eq. (36), i.e.,

$$
\begin{aligned}
& \hbar \dot{\rho}_{-1,-1}=\Delta \operatorname{Im} \rho_{1,-1} \\
& \hbar \dot{\rho}_{1,-1}=-i W_{\text {eff }}(t) \rho_{1,-1}+i \Delta\left(\frac{1}{2}-\rho_{-1,-1}\right) .
\end{aligned}
$$

This is equivalent to the nonlinear Schrödinger equation

$$
\begin{aligned}
i \hbar \dot{C}_{-1} & =\frac{1}{2} W_{\text {eff }}(t) C_{-1}-\frac{\Delta}{2} C_{1} \\
i \hbar \dot{C}_{1} & =-\frac{1}{2} W_{\text {eff }}(t) C_{1}-\frac{\Delta}{2} C_{-1}
\end{aligned}
$$

with $W_{\text {eff }}(t)=W(t)+2 J_{0}\left(1-2\left|C_{-1}\right|^{2}\right)$. It is interesting to note that if one solves Eq. (41) perturbatively in $J$ using Eq. (35) one obtains Eqs. (36) and (38). This implies 
that the MFA works well in the weak-coupling limit in our model (see Fig. 5).

In the case $N \gg 1$ one can assume that the state of the system is described by a narrow packet in the $k$-space, as is indeed the case for noninteracting particles, see Eq. (31). Then in Eq. (34) one can replace $(N-2 k-1) \Rightarrow$ $\left(N-2 k_{\max }-1\right)$ with $k_{\max }$ of Eq. (28), after which the sum over $k$ converts to $\rho_{-1,1}$ according to the definition in Eq. (33). This leads to Eq. (41). One should, however, realize that this is no more than a heuristic derivation since it was not proved that the state of a system with interaction is described by a narrow packet for $N \gg$ 1. On the other hand, the narrow-packet assumption is quite plausible since the limit $N \gg 1$ corresponds to the quasiclassical limit $S \gg 1$ of the equivalent model of Eq. (12) and the states of quasiclassical systems should be narrow packets. It is remarkable that the density-matrix equation within the MFA, Eq. (41), can be interpreted as a purely classical equation of motion for a spin vector, the Landau-Lifschitz equation (LLE). Indeed, rewriting Eq. (33) in terms of $c_{k}$ with the help of Eq. (9) and adding Eq. (14) one obtains the mapping relations

$$
\begin{aligned}
& \rho_{1,-1}=\frac{1}{N} \sum_{k=0}^{N} l_{k, k+1} c_{k+1}^{*} c_{k}=\frac{\left\langle S_{+}\right\rangle}{2 S} \\
& \rho_{-1,-1}=\frac{1}{2}\left(1-\frac{\left\langle S_{z}\right\rangle}{S}\right) .
\end{aligned}
$$

Now one can see that the DME in the MFA, Eq. (41) is equivalent to the LLE for the classical spin vector components

$$
s_{z}=\frac{\left\langle S_{z}\right\rangle}{S}, \quad s_{+}=\frac{\left\langle S_{+}\right\rangle}{S}
$$

that reads

$$
\begin{aligned}
& \hbar \dot{s}_{z}=-\Delta s_{y} \\
& \hbar \dot{s}_{+}=-i\left[W(t)+4 J(S-1 / 2) s_{z}\right] s_{+}+i \Delta s_{z} .
\end{aligned}
$$

The vector form of this LLE is

$$
\dot{\mathbf{s}}=\gamma\left[\mathbf{s} \times \mathbf{H}_{\mathrm{eff}}\right], \quad \mathbf{H}_{\mathrm{eff}}=-\frac{\partial \mathcal{H}_{\mathrm{eff}}}{\partial \mathbf{s}},
$$

where $\gamma=1 / \hbar$ and the effective classical energy is given by

$$
\mathcal{H}_{\mathrm{eff}}=-H_{z}(t) s_{z}-H_{x} s_{x}-D_{\mathrm{cl}} s_{z}^{2}
$$

with

$$
\begin{aligned}
& H_{z}(t)=W(t), \quad H_{x}=\Delta, \\
& D_{\mathrm{cl}}=D(S-1 / 2)=J(N-1) \equiv J_{0}
\end{aligned}
$$

[cf. Eqs. (12) and (13)]. The one-particle staying probability $P_{N}$ is given by the formula

$$
P_{N}(t)=\frac{1}{2}\left[1-s_{z}(t)\right]
$$

that is analogous to Eq. (14).

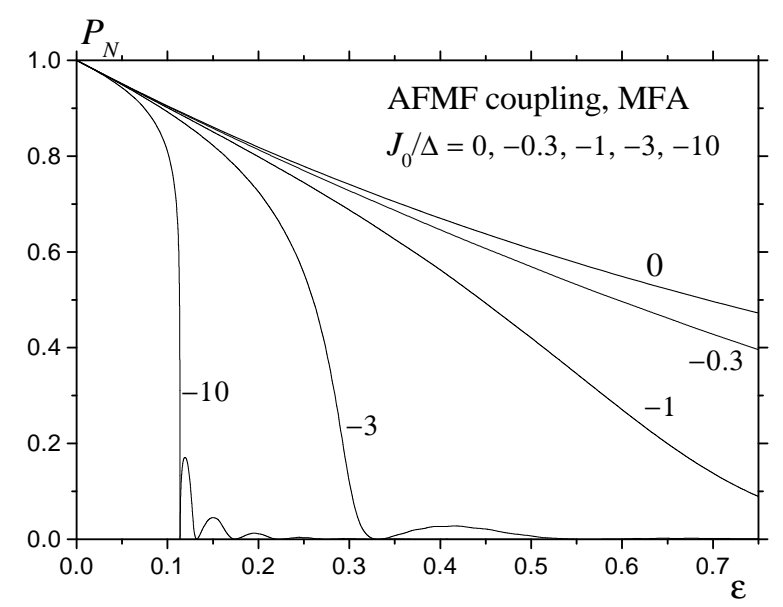

FIG. 6: The mean-field solution for the one-particle staying probability $P_{N}$ for the AFMF coupling, $J_{0} \equiv(N-1) J<0$.

Let us now analyze the mean-field solution of the LZS problem based on Eqs. (41) or (46) and compare it with the exact solution of Eq. (10). We start with the AFMF coupling, for convenience. Fig. [6 shows the dependence of the one-particle staying probability $P_{N}$ on the sweeprate parameter $\varepsilon$ for different coupling strengths $J_{0}$. The curves on the plot are qualitatively similar to those in the case of well-separated resonances, Fig. 3 and they show that AFMF interaction boosts transitions, especially for slow sweep, $\varepsilon \gg 1$. The difference is that in the MFA only the combined parameter $J_{0}=(N-1) J$ enters, whereas for well-separated resonances the results depend on $N$ only and not on $J$. Another difference is that for strong enough interaction the mean-field solution for $P_{N}$ oscillates and vanishes at some values of $\varepsilon$. Oscillations of this kind have been found in the solution of the LZS problem with nonlinear sweep $\stackrel{8.12}{=}$ As was explained in Ref. 8, oscillations arise when the sweep slows down in the vicinity of the resonance so that (in the extreme case) the particle oscillates between the two quantum states and the resulting staying probability depends on the phase of this oscillation at the end of the stay near the resonance. This is also the case for the model with AFMF interaction in the MFA.Tunneling of particles creates a molecular field that changes in the direction opposite to the sweep so that the resulting effective sweep $W_{\text {eff }}(t)$ of Eq. (40) can even become nonmonotonic (in this case the analytical method of Ref. 7 based on the linearization of the problem near the resonance and introducing an effective sweep rate breaks down). The MFA solution becomes time symmetric in the case of complete conversion, $P_{N}(\infty)=0$ that for $J_{0} / \Delta=-10$ is realized for $\varepsilon=0.1137983,0.13207,0.1741,0.2233$, etc. The corresponding time dependences of $P_{N}(t)$ and $W_{\text {eff }}(t)$ are shown in Fig. (7). Although they resemble the complete conversion solutions found in Ref. 13, there are no apparent analytical solutions for this model. The difficulty of the present mean-field model is that $W_{\text {eff }}(t)$ is not known from the beginning but is a solution of a self-consistent nonlinear problem. 

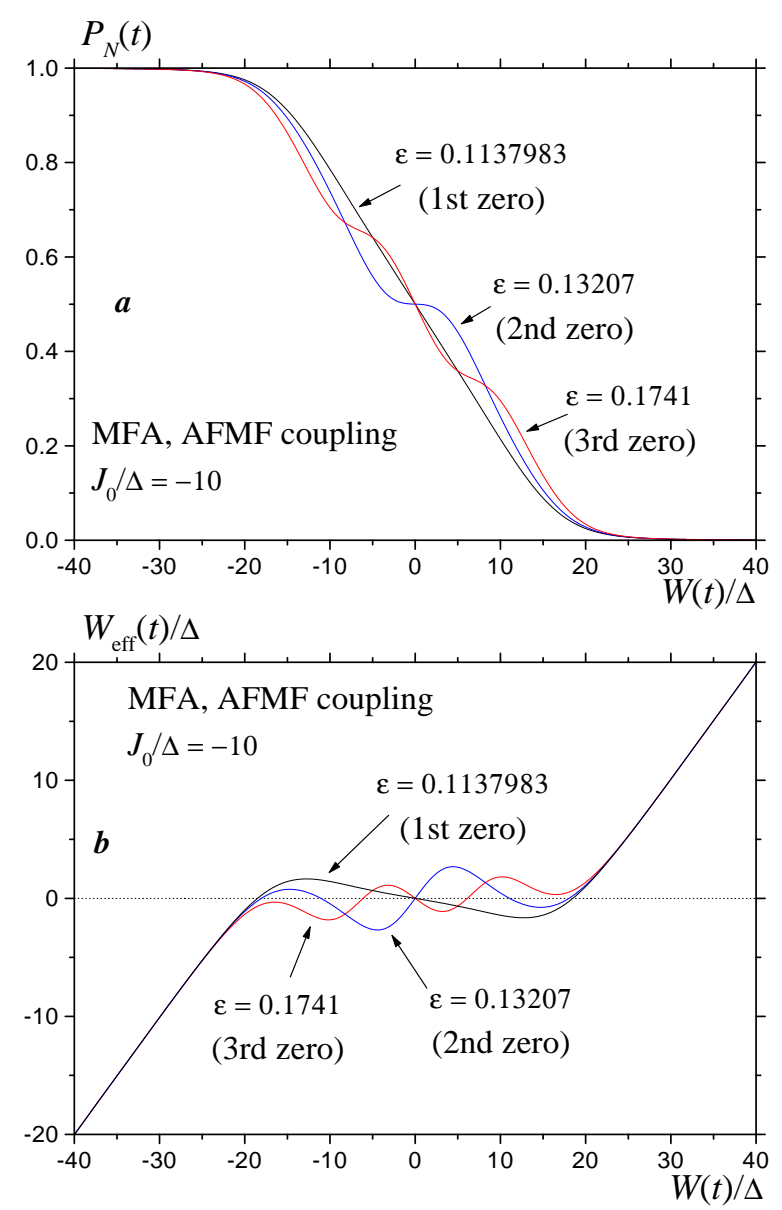

FIG. 7: $a$-Time dependence of the staying probability $P_{N}$ for the AFMF coupling $J_{0} \equiv(N-1) J=-10$ for the first three complete conversion cases, $P_{N}(\infty)=0$ (see Fig. 6). $b-$ Same for the effective sweep $W_{\text {eff }}$ of Eq. (40).

Comparison of the exact and the MFA solutions for the one-particle staying probability $P_{N}$ for the antiferromagnetic coupling $J_{0}=-10$ is shown in Fig. 8 One can see that the exact quantum solution converges to the classical mean-field solution for $N \rightarrow \infty$, although in the vicinity of the first complete-conversion point convergence is extremely slow.

Let us now turn to the ferromagnetic interaction. The mean-field solution for different interaction strengths is shown in Fig. 9 Again, qualitatively it is similar to the solution in the limit of well-separated resonances, Eq. (20) that is shown in Fig. 3. The difference is the same as for the AFMF coupling: The MFA solution depends on $J_{0} \equiv(N-1) J$ whereas Eq. (20) depends on $N$ only. Another difference is that in the slow-sweep limit the mean-field curve tends to a constant (for $J_{0} / \Delta>1 / 2$ ) whereas all quantum curves tend to zero. The latter are in fact combinations of several LZS exponentials corresponding to transitions of different orders shown in Fig. [1. by dotted arrows (see also Fig. 2). One can see, in particular, that the curve $N=2$ consist of two different exponentials, the curve $N=3$ consist of three different exponentials, etc. With increasing of $N$, however, the

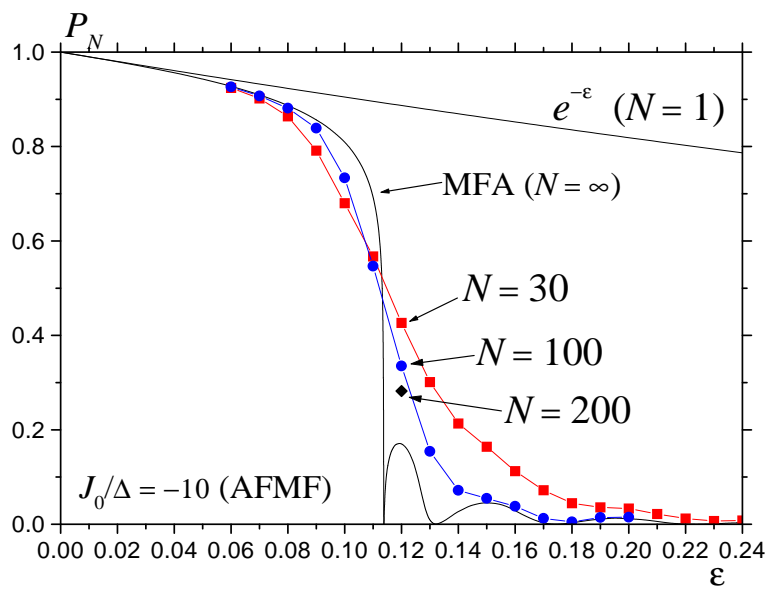

FIG. 8: Comparison of the exact quantum and the MFA solutions for the one-particle staying probability $P_{N}$ for the AFMF coupling showing partially slow convergence to the classical mean-field limit for $N \rightarrow \infty$.

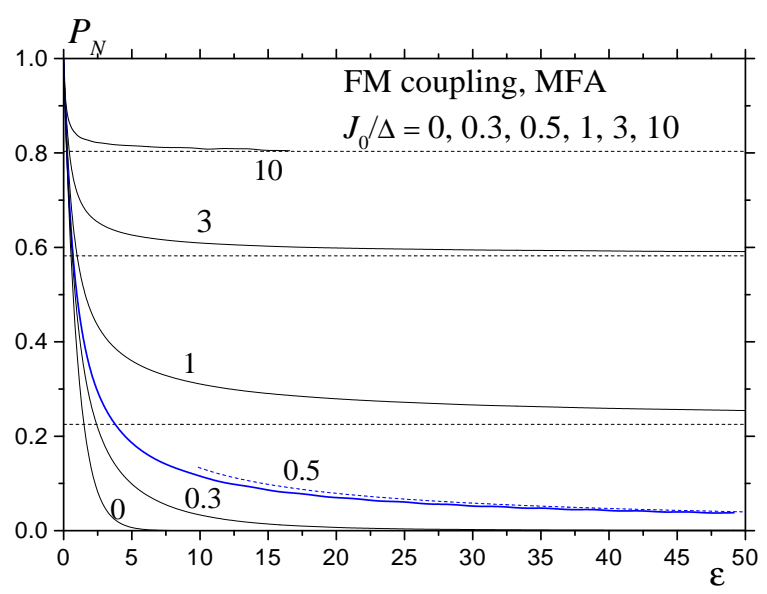

FIG. 9: The mean-field solution for the one-particle staying probability $P_{N}$ for the ferromagnetic coupling, $J_{0} \equiv(N-$ 1) $J>0$. The dashed line is the large- $\varepsilon$ asymptote of Eq. (85).

coupling $J=J_{0} /(N-1)$ decreases and transitions become not well separated. In this case dependence $P_{N}$ of $\varepsilon$ becomes a long-tale curve.

\section{SLOW SWEEP IN THE MFA}

Here we analytically consider the slow-sweep limit within the mean-field approximation, to get more insights into the mechanism that leads to suppressing transitions in the case of FM interactions (see Fig. 9) as well as into that of facilitating transitions and probabillity oscillations in the case of the antiferromagnetic frustrating interactions (see Fig. [6). 


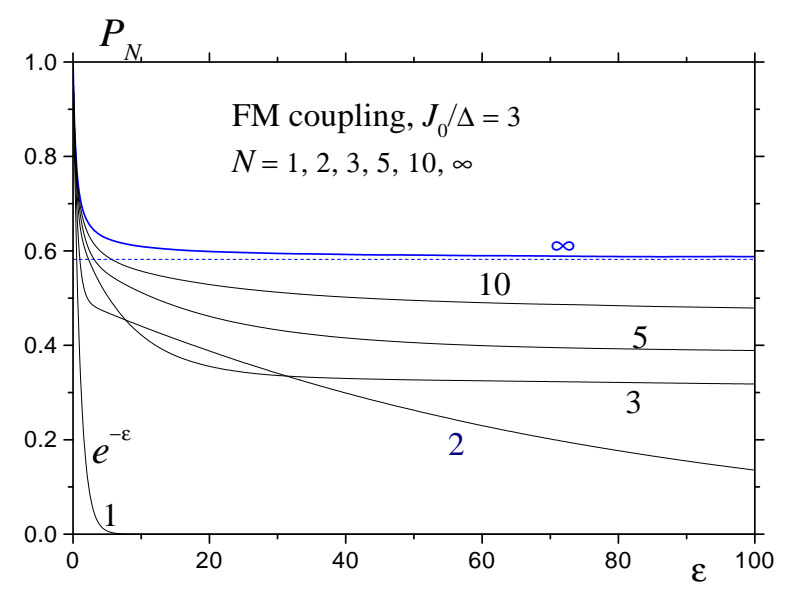

FIG. 10: Comparison of the exact quantum and the MFA solutions for the one-particle staying probability $P_{N}$ for the ferromagnetic coupling.

\section{A. Adiabatic case: Basic equations}

For the one-particle LZS problem, a convenient method of treating the slow-sweep limit is that using the adiabatic basis $\frac{8}{2}$ Its advantage is that the probability to stay on the lowest adiabatic level is at any time close to 1 , so that one can make an appropriate approximation after which the solution is obtained as a quadrature, including the case of nonlinear sweep. Direct extention of this method for the present model within the MFA is cumbersome, however, owing to the complicated selfconsistent nature of the adiabatic energy levels for a nonlinear Schrödinger equation or nonlinear density-matrix equation, Eq. (34). Fortunately, an alternative description based on the LLE, Eq. (46), supports a physically transparent extension of the method. The adiabatic basis for a Schrödinger equation corresponds to the adiabatic coordinate system for the classical problem of Eq. (46). The $z^{\prime}$ axis of this coordinate system is oriented in the direction minimizing the classical energy $\mathcal{H}_{\text {eff }}$ of Eq. (47) at any fixed time $t$. It belongs to the $x-z$ plane and it makes the angle $\theta(t)$ with the $z$ axis that satisfies the equation

$$
h_{z}(t) \sin \theta+\nu \sin \theta \cos \theta-h_{x} \cos \theta=0
$$

following from $\partial \mathcal{H}_{\mathrm{eff}} / \partial \theta=0$. Here $\nu=D_{\mathrm{cl}} /\left|D_{\mathrm{cl}}\right|=J /|J|$ and

$$
h_{x} \equiv \frac{H_{x}}{2\left|D_{\mathrm{cl}}\right|}=\frac{\Delta}{2\left|J_{0}\right|}, \quad h_{z}(t) \equiv \frac{H_{z}(t)}{2\left|D_{\mathrm{cl}}\right|}=\frac{W(t)}{2\left|J_{0}\right|} .
$$

Eq. (50) is well known in the theory of magnetism. In the AFMF case $\nu<0$, its solution is unique. For the ferromagnetic interaction $\nu>0$, Eq. (50) has two solutions corresponding to the minimum and to the maximum of $\mathcal{H}_{\text {eff }}$ in the range of reduced fields $h_{x}$ and $h_{z}$ outside the Stoner-Wohlfarth astroid 14 (see Fig. [11)

$$
h_{x}^{2 / 3}+h_{z}^{2 / 3}=1 .
$$

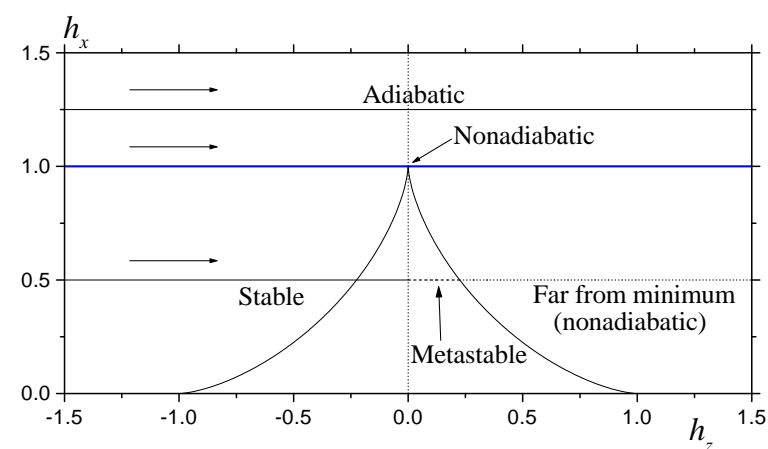

FIG. 11: Comparison of the exact quantum and the MFA solutions for the one-particle staying probability $P_{N}$ for the ferromagnetic coupling.

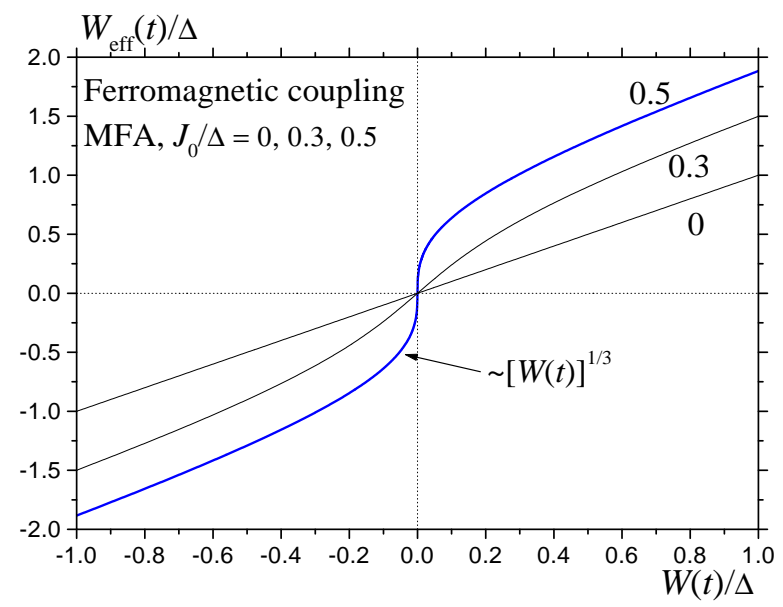

FIG. 12: Effective sweep in the adiabatic case for the ferromagnetic coupling of different strengths.

For $h_{x}$ and $h_{z}$ inside the astroid, Eq. (150) has four solutions corresponding to the stable and metastable minima as well as to the saddle point and to the maximum of $\mathcal{H}_{\text {eff }}$. The adiabatic solution corresponds to the minimum of the energy that changes as $h_{z}(t)$ is sweeped from $-\infty$ to $\infty$. For $h_{x} \geq 1$ the system follows this adiabatic solution in the whole range of $h_{z}$. In this case dependence of the effective sweep $W_{\text {eff }}$ of Eq. (40) or, here, $W_{\text {eff }}=H_{z}+2 D_{\mathrm{cl}} \cos \theta$, on the energy sweep $W$ is shown in Fig. 12 One can see that for $J_{0} / \Delta=0.5$ this dependence becomes nonanalytic. This corresponds to the horizontal line in Fig. 11] that touches the upper corner of the astroid. In contrast, for $h_{x}<1$ the adiabatic solution exists only until the crossing of the right branch of the astroid. After that the spin rotates away from the disappeared metastable state and its behavior becomes nonadiabatic. The latter case will be considered later while at first we concentrate on the adiabatic situation.

The Landau-Lifshitz equation, Eq. (46) can be rewritten in the rotating adiabatic coordinate system as follows

$$
\dot{\mathbf{s}}^{\prime}=\left[\mathbf{s}^{\prime} \times\left(\gamma \mathbf{H}_{\mathrm{eff}}^{\prime}+\boldsymbol{\Omega}\right)\right], \quad \boldsymbol{\Omega}=\dot{\theta} \mathbf{e}_{y},
$$

where $\dot{\theta}$ is time derivative of the appropriate solution of 
Eq. (50). The reduced effective field

$$
\mathbf{h}_{\mathrm{eff}} \equiv \frac{\mathbf{H}_{\mathrm{eff}}}{2\left|D_{\mathrm{cl}}\right|}=\left(h_{z}+\nu s_{z}\right) \mathbf{e}_{z}+h_{x} \mathbf{e}_{x},
$$

in the adiabatic frame is calculated as follows

$$
\begin{aligned}
\mathbf{h}_{\mathrm{eff}}^{\prime}= & \left(h_{x} \cos \theta-h_{\mathrm{eff}, z} \sin \theta\right) \mathbf{e}_{x^{\prime}} \\
& +\left(h_{x} \sin \theta+h_{\mathrm{eff}, z} \cos \theta\right) \mathbf{e}_{z^{\prime}} \\
h_{\mathrm{eff}, z}= & h_{z}+\nu\left(-s_{x^{\prime}} \sin \theta+s_{z^{\prime}} \cos \theta\right) .
\end{aligned}
$$

With the help of Eq. (50) components of $\mathbf{h}_{\text {eff }}^{\prime}$ simplify to

$$
\begin{aligned}
h_{\mathrm{eff}, x^{\prime}}= & \left(1-s_{z^{\prime}}+s_{x^{\prime}} \tan \theta\right) \nu \sin \theta \cos \theta \\
h_{\mathrm{eff}, z^{\prime}}= & h_{x} / \sin \theta-s_{x^{\prime}} \nu \sin \theta \cos \theta \\
& +\left(1-s_{z^{\prime}}\right)\left(h_{z}-h_{x} \cot \theta\right) \cos \theta .
\end{aligned}
$$

Note that if the spin is directed along the $z^{\prime}$ axis, i.e., $s_{x^{\prime}}=s_{y}=0$ and $\mathrm{s}_{z^{\prime}}=1$, one has $h_{\mathrm{eff}, x^{\prime}}=0$, that is consistent with the choice of the adiabatic coordinate system. Introducing the dimensionless sweep variables

$$
u \equiv \frac{W(t)}{\Delta}=\frac{v t}{\Delta}=\frac{h_{z}}{h_{x}}, \quad \tilde{\varepsilon} \equiv \frac{\Delta^{2}}{\hbar v}
$$

[ $\tilde{\varepsilon}$ should not be confused with $\varepsilon=(\pi / 2) \tilde{\varepsilon}$ of Eq. (3)] one can rewrite Eq. (53) in the form

$$
\frac{d \mathbf{s}^{\prime}}{d u}=\left[\mathbf{s}^{\prime} \times\left(\tilde{\varepsilon} \frac{\mathbf{h}_{\mathrm{eff}}^{\prime}}{h_{x}}+\frac{d \theta}{d u} \mathbf{e}_{y}\right)\right] .
$$

In the slow-sweep limit $\varepsilon \gg 1$ the solution of Eq. (58) is close to the adiabatic solution $s_{x^{\prime}}=s_{y}=0$ and $\mathrm{s}_{z^{\prime}}=1$. Hence one can linearize this equation near the adiabatic solution:

$$
\begin{aligned}
s_{z^{\prime}} & \Rightarrow 1, \quad h_{\mathrm{eff}, z^{\prime}} \Rightarrow h_{x} / \sin \theta \\
h_{\mathrm{eff}, x^{\prime}} & \Rightarrow s_{x^{\prime}} \nu \sin ^{2} \theta
\end{aligned}
$$

that results in

$$
\begin{aligned}
\frac{d s_{x^{\prime}}}{d u} & =\frac{\tilde{\varepsilon}}{\sin \theta} s_{y}-\frac{d \theta}{d u} \\
\frac{d s_{y}}{d u} & =-\frac{\zeta \tilde{\varepsilon}}{\sin \theta} s_{x^{\prime}},
\end{aligned}
$$

where

$$
\zeta \equiv 1-\rho \sin ^{3} \theta, \quad \rho \equiv 2 J_{0} / \Delta .
$$

The factor $\zeta$ in the second of Eqs. (60) makes spin precession elliptic for any nonzero coupling $J_{0}$, especially in the extreme case $\rho=1$. This is an important difference from the model of one tunneling particle with a nonlinear

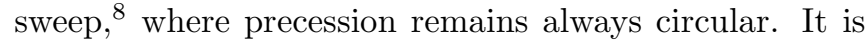
convenient to introduce

$$
\tilde{c}_{+} \equiv-\frac{1}{2}\left(\zeta^{1 / 4} s_{x^{\prime}}+i \zeta^{-1 / 4} s_{y}\right), \quad \bar{\Omega}(u) \equiv \frac{\sqrt{\zeta}}{\sin \theta}
$$

and rewrite Eqs. (60) in the form

$$
\frac{d \tilde{c}_{+}}{d u}=-i \tilde{\varepsilon} \bar{\Omega}(u) \tilde{c}_{+}+\frac{1}{2} \frac{d \theta}{d u} \zeta^{1 / 4} .
$$

This differential equation can be easily solved with the initial condition $\tilde{c}_{+}(-\infty)=0$, and the staying probability $P_{N}$ can be found from Eq. (49). Keeping in mind that at $t=\infty$ both coordinate systems coincide whereby $\theta=0$ and $a=1$, one can rewrite $P_{N} \equiv P_{N}(\infty)$ within our linearized theory as

$$
P_{N} \cong \frac{1}{4}\left[s_{x^{\prime}}^{2}(\infty)+s_{y}^{2}(\infty)\right]=\left|\tilde{c}_{+}(\infty)\right|^{2} .
$$

The final general expression for $P_{N}$ reads

$$
P_{N} \cong\left|\frac{1}{2} \int_{-\infty}^{\infty} d u \frac{d \theta}{d u} \zeta^{1 / 4} \exp [i \tilde{\varepsilon} \Phi(u)]\right|^{2},
$$

where

$$
\Phi(u) \equiv \int_{0}^{u} d u^{\prime} \bar{\Omega}\left(u^{\prime}\right) .
$$

In the absence of interaction $J=0$, our solution recovers that for the standard LZS problem in the slow-sweep limit up to the prefactor in front of a small exponential. Indeed, in this case the solution of Eq. (50) is

$$
\cos \theta=\frac{h_{z}}{\sqrt{h_{x}^{2}+h_{z}^{2}}}=\frac{u}{\sqrt{1+u^{2}}},
$$

in Eq. (61) one has $\rho=0$ and $\zeta=1$, hence

$$
\bar{\Omega}(u)=\sqrt{1+u^{2}}, \quad \frac{d \theta}{d u}=-\frac{1}{1+u^{2}}=-\frac{1}{\bar{\Omega}^{2}(u)} .
$$

One can see that in this case Eq. [63) coincides with second of Eqs. (24) of Ref. 8 and Eq. (65) coincides with Eq. (26) of Ref. 8, with $w^{\prime}(u)=1$ and $\tilde{c}_{-} \Rightarrow 1$. The latter is exactly the approximation proposed in Ref. 8 that for the standard LZS problem reproduces the wellknown result of Eq. (3) for $\varepsilon \gg 1$, however with a wrong prefactor: $P \approx(\pi / 3)^{2} e^{-\varepsilon}$. In Ref. 8 we have shown how to correct the prefactor by accurately calculating $\tilde{c}_{-}$.

\section{B. Adiabatic case: The results}

In the general case $J \neq 0$ Eq. (50) does not yield an analytical solution for $\theta(u)$. Fortunately, instead of $u$ one can use $x \equiv \cos \theta$ as the integration variable on the interval $-1 \leq x \leq 1$ and with the help of Eq. (50) express $u=h_{z} / h_{x}$ as a function of $x$. Even better is then to parametrize $x$ as $x=w / \sqrt{1+w^{2}}$, analogously to Eq. (67). This brings Eq. (65) into the explicit form

$$
P_{N} \cong\left|\frac{1}{2} \int_{-\infty}^{\infty} \frac{d w}{1+w^{2}}\left[1-\frac{\rho}{\left(1+w^{2}\right)^{3 / 2}}\right]^{1 / 4} e^{i \tilde{\varepsilon} \Phi(w)}\right|^{2},
$$

where

$$
\Phi(w)=\int_{0}^{w} d w \sqrt{1+w^{2}}\left[1-\frac{\rho}{\left(1+w^{2}\right)^{3 / 2}}\right]^{3 / 2} .
$$


In the absence of interaction, $\rho=0$, one has $w=u$ and the formulas above describe the standard LZS effect. For $\varepsilon \gg 1$, the value of $P_{N}$ is exponentially small and defined by the singularities of the integrand in the complex plane of $w$

$$
P_{N}=P_{N 0} e^{-\varepsilon \operatorname{Im} F(\rho)},
$$

where

$$
F(\rho)=\frac{4}{\pi} \int_{0}^{w_{c}} d w \sqrt{1+w^{2}}\left[1-\frac{\rho}{\left(1+w^{2}\right)^{3 / 2}}\right]^{3 / 2} .
$$

For $\rho=0$ one obtains the LZS value $F(0)=i$. For the FM coupling the relevant singularity is

$$
w_{c}=i \sqrt{1-\rho^{2 / 3}}, \quad 0<\rho<1,
$$

that corresponds to vanishing of $\bar{\Omega}(u)$ of Eq. (62) due to that of the ellipticity factor $\zeta$. For $\rho>1$, the motion of the spin becomes nonadiabatic (see Fig. 111), and this method does not apply any longer. For $0<\rho<1$ one has $\operatorname{Re} F(\rho)=0$ and the limiting forms of $\operatorname{Im} F(\rho)$ are given by

$$
\operatorname{Im} F(\rho) \cong \begin{cases}1-\frac{2}{\pi}\left(\ln \frac{32}{\rho}-1\right) \rho, & \rho \ll 1 \\ \frac{\sqrt{3}}{2 \sqrt{2}}(1-\rho)^{2}, & 1-\rho \ll 1 .\end{cases}
$$

For the AFMF coupling $\rho<0$, there is a pair of relevant singularities that also correspond to $\zeta=0$ and are given by

$$
\begin{aligned}
w_{c \pm} & =i\left(1+|\rho|^{2 / 3}+|\rho|^{4 / 3}\right)^{1 / 4} \exp \left(\mp i \varphi_{c}\right) \\
\varphi_{c} & =\frac{1}{2} \arctan \frac{\sqrt{3}|\rho|^{2 / 3}}{2+|\rho|^{2 / 3}} .
\end{aligned}
$$

Note that $\operatorname{Im}\left(w_{c \pm}\right)>1$ for $\rho<0$ and thus these singularities are further from the real axis than the LZS singularity at $w=i$. The latter, however, makes no contribution since, as can be easily checked, $\operatorname{Im} F(\rho)=\infty$ for $w_{c}=i$ and $\rho<0$. Analytical calculation of the limiting forms of $F(\rho)$ is more cumbersome for the antiferromagnetic coupling. For $|\rho| \ll 1$ one obtains

$$
\operatorname{Re} F(\rho) \cong 2|\rho|, \quad \operatorname{Im} F(\rho) \cong 1-\frac{2}{\pi}\left(\ln \frac{32}{|\rho|}-1\right) \rho .
$$

Note that $\operatorname{Im} F(\rho)$ is given by the same formula for $|\rho| \ll$ 1 and both signs of $\rho$. In the limit $|\rho| \gg 1$ the result has the form

$$
\begin{aligned}
F(\rho) \cong & \frac{2}{3 \sqrt{\pi}} \frac{\Gamma(1 / 4)}{\Gamma(3 / 4)}\left(|\rho|^{3 / 2}-\frac{1}{4}|\rho|^{5 / 6}\right) \\
& +\frac{\sqrt{\pi}(\sqrt{3}+i)}{\Gamma(5 / 3) \Gamma(11 / 6)}|\rho|^{2 / 3} \\
\simeq & 1.1|\rho|^{3 / 2}-0.3|\rho|^{5 / 6}+(3.6+2.1 i)|\rho|^{2 / 3}(77)
\end{aligned}
$$

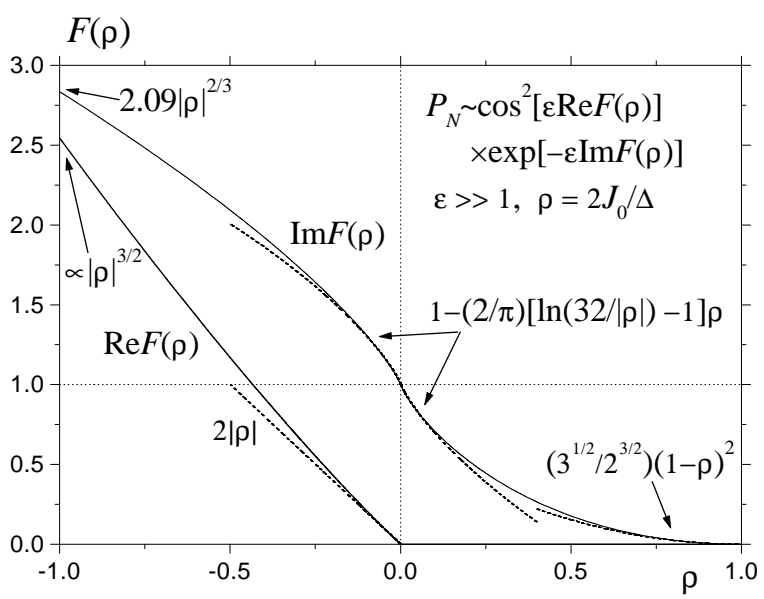

FIG. 13: Function $F(\rho)$ of Eqs. (71) and (72).

The real and imaginary parts of $F(\rho)$ numerically calculated from Eq. (72), as well as the analytical limiting forms, are shown in Fig. 13.

The prefactor $P_{N 0}$ in Eq. (71) is determined for $\varepsilon \gg 1$ by a close vicinity of the singularities $w_{c}$. For the ferromagnetic coupling the result is

$$
P_{N 0} \cong \frac{\pi^{2}}{15 \varepsilon \rho \sqrt{1-\rho^{2 / 3}}}, \quad 0<\rho<1 .
$$

For the AFMF coupling $\rho<0$ interference of the two contributions from $w_{c+}$ and $w_{c-}$ of Eq. (75) results in the oscillating prefactor

$$
P_{N 0} \cong \frac{\pi^{2}}{15 \varepsilon|\rho|\left|w_{c}\right|} \cos ^{2}\left[\frac{\varepsilon}{2} \operatorname{Re} F(\rho)\right], \quad \rho<0 .
$$

Oscillating prefactors of such a kind take place for one tunneling particle if the sweep is nonlinear and decelerating in the vicinity of the resonance $\stackrel{8}{\underline{8}}$ Here effective nonlinearity of this type occurs because of the negative coupling between tunneling particles, even for the linear sweep.

Both Eqs. (78) and (79) break down in the linit $\rho \rightarrow 0$ since different singularities come close to each other. One could work out the crossover from Eqs. (78) and (79) to the value $P_{N 0}=(\pi / 3)^{2}$ (see Ref. [8) at $\rho=0$ that takes place in a narrow region $|\rho| \varepsilon \sim 1$. This makes no sence, however, since the result $P_{N 0}=\pi / 3$ differs from the exact LZS prefactor $P_{0}=1$ and it has to be improved by taking into account nonlinear terms dropped during the derivation of Eq. 63). The corresponding procedure for $\rho=0$ is described in Ref. 8. In the present case $\rho \neq 0$ this would be too involved and we don't try to do it. It is clear that the logarithmic singularity of the exponent of Eq. (76) should ve compensated for by the singularity of the prefactor so that the staying probability $P_{N}$ behaves linearly in $\rho$ near $\rho=0$ [see Eq. (39)].

Let us now consider the case $1-\rho \ll 1$ in more detail since the prefactor $P_{N 0}$ of Eq. (78) diverges at $\rho \rightarrow 1$. Here one has $\operatorname{Im} F(\rho) \ll 1$, and the exponential decrease of $P_{N}$ is very slow. In this region according to Eq. (73) 
the integral in Eq. (69) is dominated by small $w$ and it can be simplified to

$$
\begin{aligned}
P_{N} & \cong \frac{2}{3} \delta^{3 / 2}\left|\frac{1}{2} \int_{-\infty}^{\infty} d t\left(1+t^{2}\right)^{1 / 4} e^{i a \widetilde{\Phi}(t)}\right|^{2} \\
\widetilde{\Phi}(t) & =\int_{0}^{t} d t^{\prime}\left(1+t^{\prime 2}\right)^{3 / 2}
\end{aligned}
$$

where

$$
\delta \equiv 1-\rho \ll 1, \quad t \equiv \sqrt{\frac{3}{2 \delta}} w, \quad a \equiv \sqrt{\frac{2}{3}} \delta^{2} \tilde{\varepsilon} .
$$

It is convenient to compute the integral in Eq. (80) by shifting the integration contour by $i$ to suppress oscillations of the integrand, i.e., to parametrize $t=i+z$, $-\infty<z<\infty$. With $\widetilde{\Phi}(i)=i 3 \pi / 8$ this yields

$$
P_{N} \cong P_{N 0} e^{-a \operatorname{Im} \widetilde{\Phi}(i)}=P_{N 0} \exp \left(-\frac{3 \pi}{8} a\right),
$$

where the exponent coincides with the previously obtained $[\sqrt{3} /(2 \sqrt{2})] \delta^{2} \varepsilon$ [see second line of Eq. (744)]. The prefactor $P_{N 0}$ can be calculated analytically for $a \gg 1$ and $a \ll 1$. For $a \gg 1$ we need the small- $z$ expansions

$$
\begin{aligned}
& \delta \widetilde{\Phi}(z) \equiv \widetilde{\Phi}(i+z)-\widetilde{\Phi}(i) \cong \frac{4}{5}(-1+i) z^{5 / 2} \\
& {\left[1+(i+z)^{2}\right]^{1 / 4} \cong(-1)^{1 / 8}(2 z)^{1 / 4} .}
\end{aligned}
$$

Values of these functions for $z<0$ are obtained from $(-1)^{5 / 2}=-i$ and $(-1)^{1 / 4}=(1-i) / \sqrt{2}$. After that calculation in Eq. (80) yields

$$
P_{N 0} \cong \frac{2 \pi}{15} \frac{\delta^{3 / 2}}{a}, \quad a \gg 1
$$

that is a limiting form of Eq. (78). In the opposite limit $a \ll 1$, the integral in Eq. (80) is dominated by large $t$, so that one can use $\widetilde{\Phi}(t) \cong t^{4} / 4$ and $\left(1+t^{2}\right)^{1 / 4} \cong t^{1 / 2}$. This yields

$$
P_{N 0} \cong \cos ^{2}\left(\frac{3 \pi}{16}\right) \frac{\Gamma^{2}(3 / 8)}{6 \sqrt{2}} \frac{\delta^{3 / 2}}{a^{3 / 4}} \simeq \frac{0.747837}{\varepsilon^{3 / 4}}, \quad \rho \rightarrow 1 .
$$

It is convenient to represent $P_{N 0}(a, \delta)$ in the whole range of $a$ with the help of the crossover function $f(a)$ according to

$$
\begin{aligned}
& P_{N 0}(a, \delta)=\frac{2 \pi}{15} \frac{\delta^{3 / 2}}{a} f(a) \\
& f(a) \cong \begin{cases}1, & a \gg 1 \\
1.09294 a^{1 / 4}, & a \ll 1 .\end{cases}
\end{aligned}
$$

Numerically computed function $f(a)$ is shown in Fig. (14).

\section{Strong ferromagnetic interactions}

For stronger ferromagnetic interactions, $\rho>1$, the adiabatic approximation breaks down since the spin approximately follows the initial energy minimum that becomes

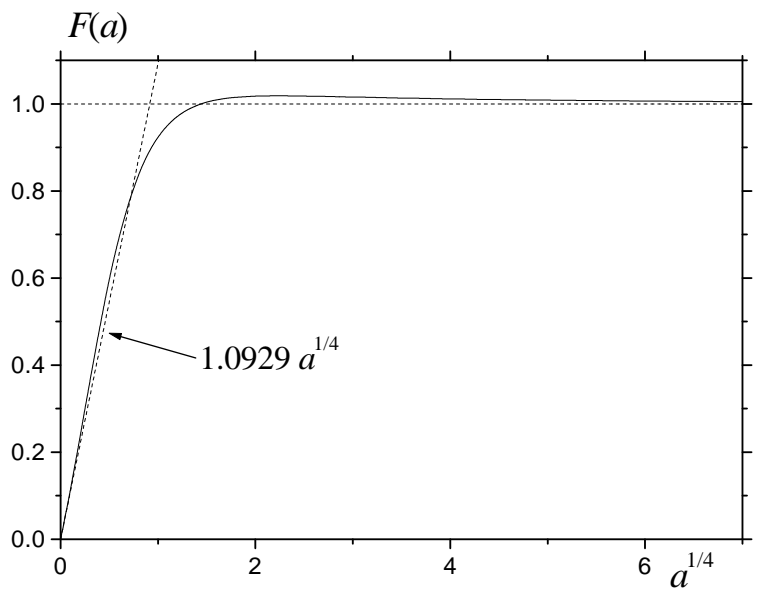

FIG. 14: Scaling function $f(a)$ of Eq. 86

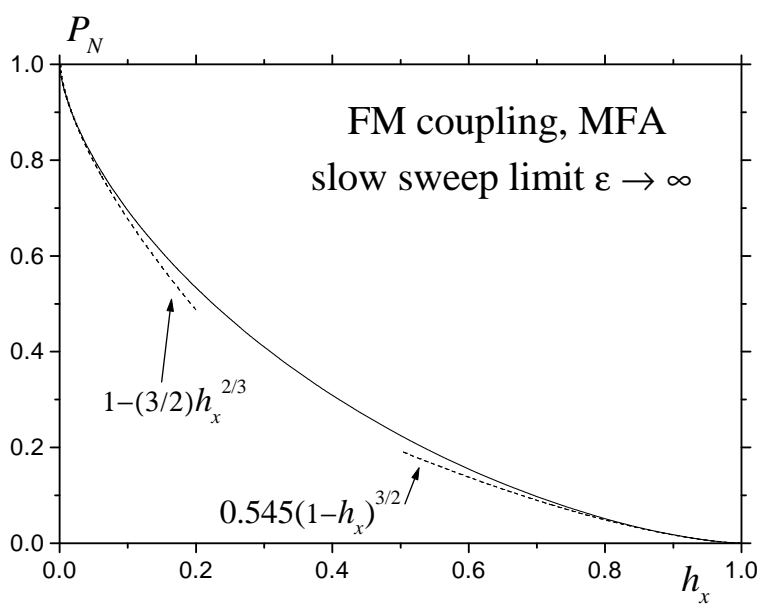

FIG. 15: $P_{N}$ in the slow-sweep limit $\varepsilon \rightarrow \infty$ vs $h_{x}=1 / \rho=$ $\Delta /\left(2 J_{0}\right)$.

unstable at some $h_{z}(t)>0$ (see Fig. 11) and than it performes a large motion that does not approach the new stable energy minimum and thus cannot be linearized around it. While the problem becomes much more complicated in this case, one can still find analytically $\lim _{\varepsilon \rightarrow \infty} P_{N}$ that is nonzero. To this end, one can represent the LLE for the spin, Eq. (46), in the Hamiltonian form in terms of the canonical angle variables $\{\cos \theta, \varphi\} \Leftrightarrow\{p, q\}$ (in the arbitrary frame)

$$
\frac{d}{d t} \cos \theta=-\frac{\partial \mathcal{H}}{\partial \varphi}, \quad \frac{d}{d t} \varphi=\frac{\partial \mathcal{H}}{\partial \cos \theta}
$$

and use conservation of the action

$$
\mathcal{S}=\oint p d q=\oint \cos \theta d \varphi
$$

over the period of motion for very slowly changing parameters of the system [here $\left.h_{z}(t)\right]$. Since in the final state $h_{z} \rightarrow \infty$ the spin precesses around the $z$ axis with a constant value of $s_{z}(\infty)=\cos [\theta(\infty)]$ that is related to the staying probability $P_{N}$ by Eq. (49) and the corresponding action is simply $\mathcal{S}(\infty)=2 \pi \cos \theta(\infty)$, one 
obtains

$$
P_{N}=\frac{1}{2}\left(1-\frac{1}{2 \pi} \mathcal{S}\right), \quad \varepsilon \rightarrow \infty,
$$

where $\mathcal{S}$ is the action over the trajectory that starts from the metastable energy minimum that is on the verge of being unstable

$$
s_{z s}=-\left(1-h_{x}^{2 / 3}\right)^{1 / 2}, \quad s_{x s}=h_{x}^{1 / 3}, \quad s_{y s}=0 .
$$

This trajectory can be found from the conservation of energy,

$$
-s_{z}^{2}-2\left(1-h_{x}^{2 / 3}\right)^{3 / 2} s_{z}-2 h_{x} s_{x}=1-3 h_{x}^{2 / 3} .
$$

Analysis shows that in the range $0<h_{x}<3 \sqrt{3} / 8$ this trajectory encircles the $z$ axis, thus the $z$ axis can be used as the polar axis $\left(s_{z}=\cos \theta, s_{x}=\sin \theta \cos \varphi, s_{y}=\right.$ $\sin \theta \sin \varphi)$ to compute $\mathcal{S}$. In the overlapping range $1 / 8<$ $h_{x}<1$ the trajectory encircles the $x$ axis that can be used as the polar axis $\left(s_{x}=\cos \theta, s_{z}=-\sin \theta \cos \varphi\right.$, $\left.s_{y}=\sin \theta \sin \varphi\right)$. With these choices, $\cos \theta$ can be found numerically from Eq. (91) as a function of $\varphi$ and the action $\mathcal{S}$ can be computed from Eq. (88). The result for $P_{N}$ vs $h_{x}$ is shown in Fig. 15 with asymptotes

$$
P_{N} \cong \begin{cases}1-(3 / 2) h_{x}^{2 / 3}, & h_{x} \ll 1 \\ 0.544861\left(1-h_{x}\right)^{3 / 2}, & 1-h_{x} \ll 1\end{cases}
$$

\section{DISCUSSION}

The simplified model of interacting tunneling particles that was considered above allows to quantify the influence of interaction on the Landau-Zener-Stueckelberg staying probability $P$. It was done here by numerically solving the problem for up to $N=200$ interacting particles as well as using a number of analytical and half-analytical approaches, including the mean-field limit $N \rightarrow \infty$.

In accord with physical expectations and considerations of the exact levels of the system in Fig. 17, the ferromagnetic coupling tends to suppress LZS transitions to another bare energy level (i.e., the state on the other side of the energy barrier), in agreement with Ref. 7. For $N \rightarrow \infty$ there is a critical value of the coupling above which the staying probability $P$ does not go to zero in the slow-sweep limit $\varepsilon \rightarrow \infty$, in contrast with the standard LZS case. For finite $N$ the dependence of $P$ on $\varepsilon$ is a sum of many exponentials with greatly differing relaxation rates that is very slow approaching zero (see Fig. 10). The same should be the case for more realistic interactions of the ferromagnetic type.

The negative coupling in our model (that corresponds to the antiferromagnetic frustrating coupling) tends to boost the LZS transitions. In the limit $N \rightarrow \infty$ the staying probability $P$ even turns to zero at finite values of the sweep rate while the effective sweep rate becomes an odd function of time with retardation in the resonancecrossing region (see Fig. (7). On the other hand, models with more realistic antiferromagnetic interactions such as the nearest-neighbor interaction have the energy-level scheme strongly differing from that shown in Fig. 1p. Preliminary results show that such interactions tend to hamper LZS transitions instead of boosting it, although not to such an extent as ferromagnetic interactions.

Theoretically the model considered here is interesting since it maps on the problem of a single large spin $S=$ $N / 2$ and thus the mean-field limit $N \rightarrow \infty$ corresponds to the classical limit $S \rightarrow \infty$ for the large spin. It would be very interesting to study deviations from the meanfield solutions for large but finite $N$. These deviations can be very large, as can be seen in Fig. 8 in the region $\varepsilon \simeq 0.11$. How quantitatively well does the MFA work for model systems with more complicated interactions and for realistic systems remains unclear, and it is an interesting topic for further work.

\section{ACKNOWLEDGMENTS}

Many useful discussions with Rolf Schilling are greatfully acknowledged.
1 L. D. Landau, Phys. Z. Sowjetunion 2, 46 (1932).

2 C. Zener, Proc. R. Soc. London A 137, 696 (1932).

3 E. C. G. Stueckelberg, Helv. Phys. Acta 5, 369 (1932).

4 D. S. F. Crothers and J. G Huges, J. Phys. B 10, L557 (1977).

${ }^{5}$ W. Wernsdorfer and R. Sessoli, Science 284, 133 (1999).

${ }^{6}$ W. Wernsdorfer, R. Sessoli, A. Caneshi, D. Gatteschi, and A. Cornia, Europhys. Lett. 50, 552 (2000).

7 A. Hams, H. De Raedt, S. Miyashita, and K. Saito, Phys. Rev. B 62, 13880 (2000).

8 D. A. Garanin and R. Schilling, Phys. Rev. B 66, 174438 (2002).

9 V. M. Akulin and W. P. Schleich, Phys. Rev. A 46, 4110
(1992)

10 V. V. Dobrovitski and A. K. Zvezdin, Europhys. Lett. 38, 377 (1997).

11 D. A. Garanin, J. Phys. A 24, L61 (1991).

12 Y. Teranishi and H. Nakamura, J. Chem. Phys. 107, 1904 (1997).

13 D. A. Garanin and R. Schilling, Europhys. Lett. 59, 7 (2002).

14 E. C. Stoner and E. P. Wohlfarth, Philos. Trans. R. Soc. London, Ser. A 240, 599 (1948); IEEE Trans. Magn. MAG-27, 3475 (1991). 


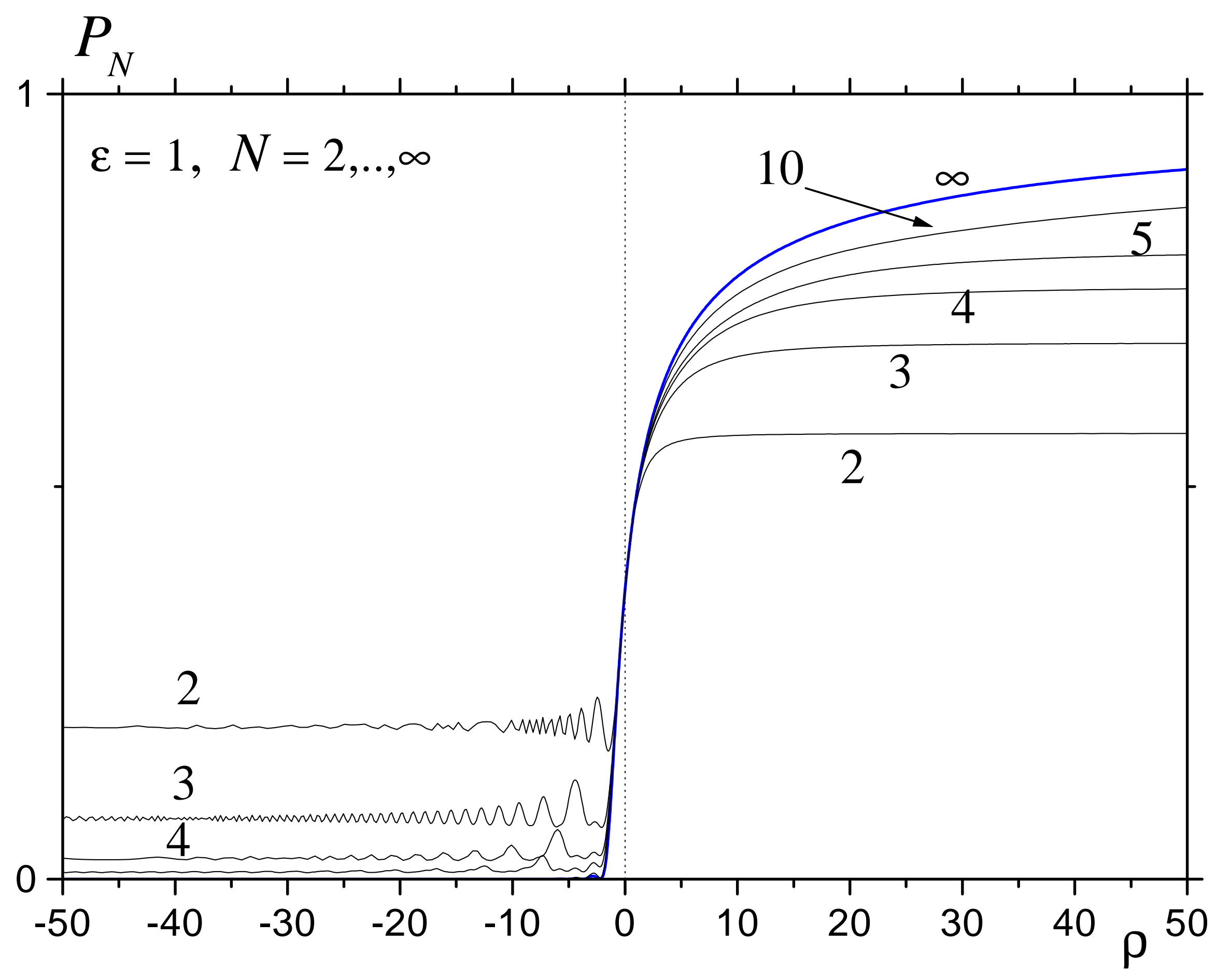

